\title{
Numerical Procedures to determine Potential Distribution from Electronic Field Vectors observed in Differential Phase Contrast (DPC) imaging
}

\author{
Akimitsu Ishizuka ${ }^{1}$, Masaaki Oka ${ }^{1}$, Kazuo Ishizuka ${ }^{1}$, Takehito Seki ${ }^{2}$ and Naoya Shibata ${ }^{2}$ \\ 1. HREM Research Inc., 14-48 Matsukazedai, Higashimatsuyama, Saitama, Japan \\ 2. Institute of Engineering Innovation, The University of Tokyo, Yayoi 2-11-16, Bunkyo, Japan
}

Recently, the differential phase contrast (DPC) imaging in STEM becomes a hot topic, since then the possibility of the DPC imaging at atomic resolution has been demonstrated [1]. The idea of the DPC imaging in STEM was proposed by Dekker and de Lang [2] and mainly used for a study of magnetic material in a medium resolution [3]. Müler et al. showed that the expectation value of the momentum transfer, which equals to a center of mass (COM) of the scattering distribution, is proportional to an electric field [4]. Thus, the COM signal corresponds to the DPC signal from the first moment detector that was discussed by Waddell and Chapman [5]. For a pure phase object the COM signal is proportional to a gradient of the object phase. Thus, the DPC experiment opens up the possibility to observe an electrostatic potential of a sample, since the object phase is proportional to a projected electric potential.

We may note that we have to solve the object phase that satisfies two COM signals: $\partial \phi(x y) / \partial x=I_{x}(x y)$ and $\partial \phi(x y) / \partial y=I_{y}(x y)$. Close et al. proposed the formula for the phase by combining the two DPC signals in Fourier space [5]:

$$
2 \pi i\left(k_{x}+i k_{y}\right) F T[\phi(x y)]=F T\left[I_{x}(x y)\right]+i F T\left[I_{y}(x y)\right] \text {. }
$$

On the other hand, we may note that $\nabla^{2} \phi(x y)=\nabla \cdot \nabla \phi(x y)=\nabla_{x} I_{x}(x y)+\nabla_{y} I_{y}(x y)$. Lazic et al. proposed the formula corresponding the Poisson equation for the phase in Fourier space [6]:

$$
2 \pi i\left(k_{x}^{2}+k_{x}^{2}\right) F T[\phi(x y)]=k_{x} F T\left[I_{x}(x y)\right]+k_{y} F T\left[I_{y}(x y)\right]
$$

We may note that Eq. (1) becomes identical to Eq. (2) only when $k_{y} F T\left[I_{x}(x y)\right]=k_{x} F T\left[I_{y}(x y)\right]$. This is equivalent to the integrability condition in real space, $\partial I_{x}(x y) / \partial y=\partial I_{y}(x y) / \partial x$, which is the necessary condition to find the object phase. In other words, Eq. (1) will give the solution that does not satisfy the integrability condition. The solution based on Eq. (1) or (2) is usually obtained by using fast Fourier transform (FFT). However, the FFT assumes the periodic boundary condition that will introduce an unwanted background to the solution of Eq. (2) (the Poisson equation in Fourier space).

Although the Poisson equation requires the boundary condition (say, Dirichlet or Neumann condition), in general we cannot measure the boundary values of an unknown solution. However, in our case our observables are not the Laplacian, but the differentials (COM signals) of the solution (electrostatic potential). Thus, we can use the Neumann boundary condition that is the differentials of the solution perpendicular to the boundary. In this case, the eigenfunction is cosine, and we can use the discrete cosine transform (DCT) instead of the solver based on the FFT, which assumes the periodic boundary condition. Figure 1 compares the results obtained with the DCT and FFT. Here, we try to retrieve the original model data from the two noise-added derivatives calculated along $\mathrm{x}$ - and $\mathrm{y}$-directions of the model (Fig. 2a). The size of the noise is $10 \%$ of the full range of each derivative.

We have also developed the real-time routine that directly integrates the observed two COM signals in real space, and updates the electrostatic potential (phase) map during the progress of the scan. In 
principle, the original function will be uniquely determined by an integral of the differentials of the function on an arbitrary path. Thus, if the signal has no noise, we can easily estimate the original function over a simple path. However, when the noise is exist, the integrals on different paths give different values. Thus, we devised a real-time routine that estimates a reliable value by integrating over many paths during the progress of the scan. Figure 2 shows the results obtained by a simple routine and the involved routine. Here, the size of the noise is also $10 \%$ of the range of each derivative.

\section{References:}

[1] Shibata N, Findlay S.D., et al. Nat. Phys. 8, 611 (2012).

[2] Dekkers N. H. and de Lang H. Optik 41, 452 (1974).

[3] Chapman J.N., Matson P.E., Waddell E.M. et al. Utramicroscopy 3, 203 (1978).

[4] Müller K, Krause FF et al., Nat. Commun. 5, 5653 (2014).

[5] Waddell E.M. and Chapman J.N. Optik 54, 83 (1979).

[6] Close R., Chen Z., Sbibata N. and Findlay S.D. Utramicroscopy 159, 124 (2015)

[7] Lazic I., Bosch E.G.T and Lazar S, Utramicroscopy 160, 265 (2016).

[8] We thank Y. Kohno from JEOL Ltd. for his helpful contribution to implement this method into realtime imaging software. A part of this study was supported by SENTAN, JST.
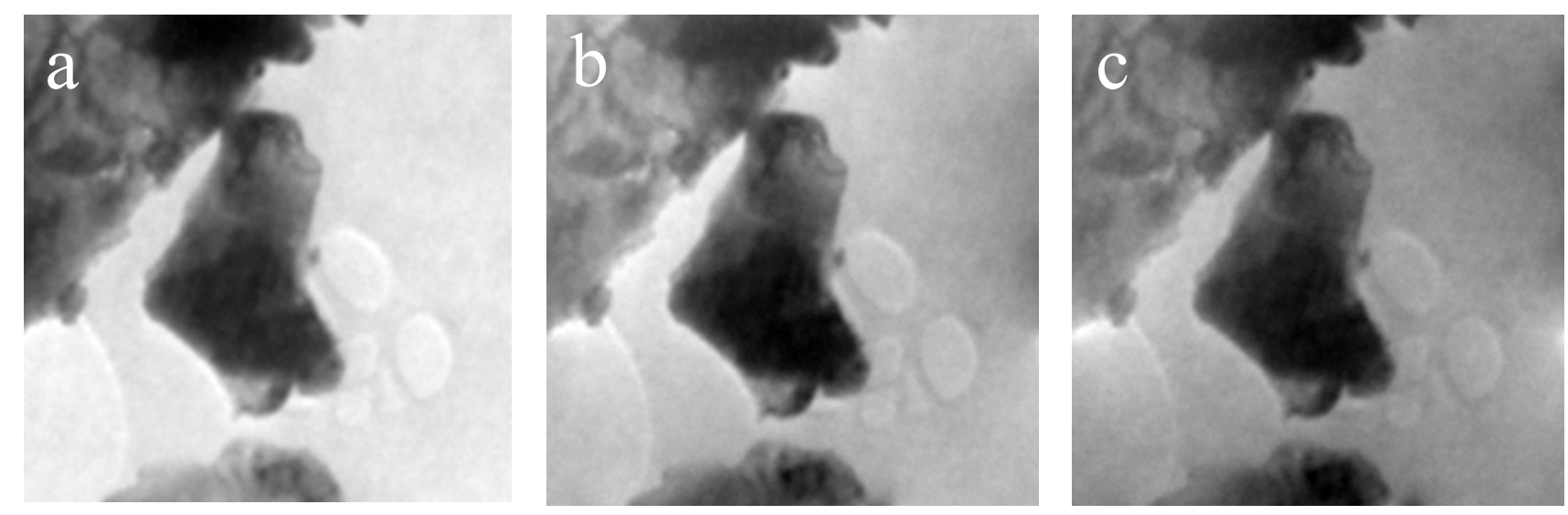

Figure 1. Retrieved maps obtained from the $x$ - and y-derivatives using (a) DCT, (b) FFT based on Eq. (2) and (c) FFT based on Eq. (1). (see Fig. 2a for the original data)
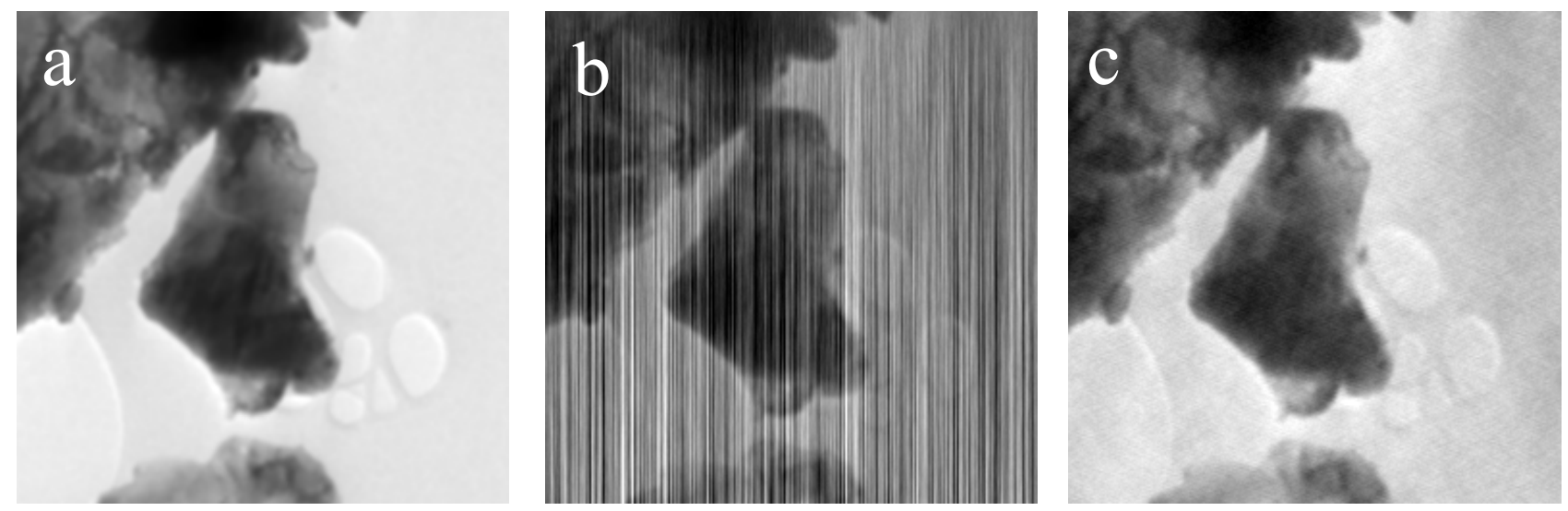

Figure 2. Real-time integration from the $x$ - and y-derivatives. (a) Original model data. Retrieved maps obtained by (b) the simple routine that integrates along the vertical direction and (c) the new routine that integrates on many integration paths. 